\title{
12 Control of energy metabolism in the brain
}

The brain regulates energy expenditure by means of hunger and satiety. The hypothalamus, a part of the diencephalon, plays a key role in this highly complex process (Vaag 2009, Nguyen et al. 2011). The hormones neuropeptide Y (NPY) and Agoutirelated protein (AGRP) stimulate appetite and reduce energy consumption in the basal metabolic rate. Antagonists of NPY and AGRP are the $\boldsymbol{\alpha}$-melanocyte-stimulating hormone ( $\boldsymbol{\alpha}-\mathrm{MSH})$ as well as the cocaine and amphetamine-regulated transcript (CART). Both curb appetite and increase energy consumption. NPY/AGRP thus act like a gas pedal on our appetite and $\alpha$-MSH/CART like a brake.

These systems initially mutually inhibit each other, while normal glucose levels act to regulate these processes. Declining glucose concentrations coupled with insufficient dietary intake, however, cancel out the inhibitory effect of the aMSH/CART cell group. The now predominantly NPY/AGRP system stimulates the production of orexins $\mathbf{A}$ and B, which trigger hunger in the lateral hypothalamus. In addition, these activate the "wake" function of the brain. After all, one has to be awake to want to consume or, as was imperative in former times, go out and forage for food. After satiety has been reached, the glucose molecules now present at higher concentrations displace the orexins of their receptors. The appetite diminishes, the person gets tired and can fall asleep better.

The insidious trap in this context, however, is today's exaggerated consumption of pure sugar, e. $g$. reflected in the frequent imbibing of soft drinks, which many start early in childhood ( $\downarrow$ Chapter 45 ). Indeed, only half of normal granulated sugar con- sists of glucose. The other half is fructose. Although fructose delivers the same number of calories as glucose, it does not act like a brake to intervene in the signaling controls of energy metabolism like glucose does ( $\triangleright$ Chapter 14 ). That is why these sugar-sweetened beverages can very rapidly cause fat to accumulate in the body (Caprio 2012, de Ruyter et al. 2012, Te Morenga et al. 2013, Page et al. 2013).

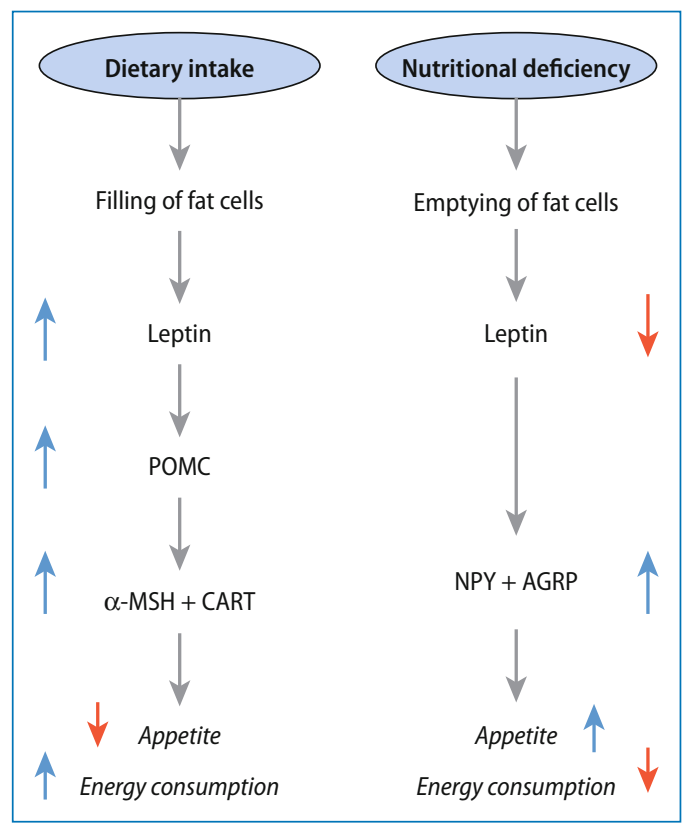

- Fig. 12.1 Regulation of appetite and energy expenditure. POMC Proopiomelanocortin, red arrow reduction, blue arrow increase 